\title{
The apparent longitude distribution of solar flares
}

\author{
A. J. Conway ${ }^{1}$ and S. A. Matthews ${ }^{2}$ \\ ${ }^{1}$ Dept. of Physics \& Astronomy, The Open University, Milton Keynes MK7 6AA, UK \\ ${ }^{2}$ Mullard Space Science Laboratory, University College London, Holmbury St. Mary, Dorking, Surrey RH5 6NT, UK
}

Received 8 August 2002 / Accepted 14 February 2003

\begin{abstract}
The distribution of solar flares in solar disk longitude averaged over many solar rotations must be uniform. However, heliographic longitude distributions constructed from observed flare locations are obviously not uniform. In the wavelength ranges we consider (hard and soft X-rays and $\mathrm{H} \alpha$ ), where the emission is presumed to be nearly isotropic, we find a wavelengthdependent bias such that fewer flares than expected occur near the limb. We can explain this bias in terms of the method of locating the flare (from $\mathrm{H} \alpha$ ) and the flare magnitude and duration. This work has implications that are relevant to measuring directivity in emission processes and also the possibility of identifying limb flares by statistical means in catalogues rather than by the time-consuming inspection of images.
\end{abstract}

Key words. Sun: magnetic fields - Sun: flares - methods: statistical - Sun: activity

\section{Introduction}

On timescales of a solar rotation there is certainly some structure in the longitude distribution of solar activity. In some cases such features, active regions or active solar longitudes, can persist for several rotations (e.g. Bumba et al. 2000). Ozguc and Altas 1996 constructed co-rotating (i.e. "fixed" to some part of the Sun's surface) longitude histograms of solar flares that exhibit evidence for active co-rotating longitudes in data spanning a solar cycle. However, averaging the heliographic (i.e. relative to the central meridian on the disc as viewed from the Earth) longitude distribution of flares over many solar rotations will "wash out" this structure, if only because of our changing viewpoint from the Earth in its orbit. The heliographic longitude distribution is therefore expected to be uniform.

Kosugi 1985 showed that the observed distribution for solar radio bursts is biased away from the limb: fewer bursts were found at longitudes near $90^{\circ}$ east or west than would be expected for a uniform distribution. It was shown that this bias was not caused by directivity of the radio emission but instead arose from the method of using $\mathrm{H} \alpha$ images to identify the location of the flare. The sample was biased because flares near the limb are more likely to be missed in $\mathrm{H} \alpha$ images because of foreshortening.

The distribution of solar flares emitting $\gamma$-rays (Vestrand et al. 1986) is clearly concentrated near the limb. This is explained by the heavily forward-beamed directivity of relativistic electrons (Petrosian 1985) that are moving towards the solar surface. More direct evidence of directivity has also been found by Kane et al. (1988) and McTiernan \& Petrosian (1990) using

Send offprint requests to: A. J. Conway, e-mail: a.j.conway@open.ac.uk stereoscopic observations of flares in a $100 \mathrm{keV}$ to $1000 \mathrm{keV}$ energy range. At lower hard X-ray energies, however, it is quite possible that the bias away from the limb is masking some mild directivity. Such underestimation of directivity of HXR radiation will lead to the underestimation of the range of pitch angles present in the electron beam (Petrosian 1985). At present, observations of directivity are the only method of probing pitch angle distributions and so can place important constraints on particle acceleration models (e.g. see Miller et al. 1997).

Flares occurring on or near the solar limb give us the clearest perspective on the structure of coronal flare emissions. Specifically, relatively faint, high energy emission in the corona, e.g. the 13th January 1992 flare - the "Masuda" flare reported by Masuda 1996, is widely believed to be the "smoking gun" of solar flares, being close to or even marking the site of primary energy release. Observations where the lower parts of the solar atmosphere are occulted by the limb are of particular interest (Kane 1979) in placing primary energy release in the corona. Such events show power laws that persist down to energies as low as $5 \mathrm{keV}$ telling us that low energy electrons are present, which, even at low coronal densities, could not have propagated far from their acceleration site. Despite their importance, limb occulted flares can be difficult to identify in catalogues because they often show little or no optical counterpart. Although they can be identified from instruments that readily detect low lying coronal emission (TRACE, Yohkoh Hard and Soft X-ray Telescopes), interesting limb flares may escape notice if such instruments are in spacecraft night, the South Atlantic Anomaly or are pointed to a different region of the solar disc. For Hard X-rays the indirect imaging involved might mean that the source location is uncertain until a preliminary image reconstruction has been performed. Also, the 
BATSE instrument on CGRO detected hard X-ray bursts that had no associated location because there was no optical counterpart and/or there were no Yohkoh observations. For all these reasons, catalogues of flares often give only the $\mathrm{H} \alpha$ position of a flare. With observed flares numbering in the tens of thousands per solar cycle we may be missing many small, but interesting (the Masuda flare was only M2.0) flares that occurred at the limb. Clearly, understanding and correcting for the $\mathrm{H} \alpha$ bias could lead to identification of more of these valuable limb flares, which was in fact the original motivation for the work presented in this paper.

In Sect. 2 we present the distributions of solar flare disk longitudes for $\mathrm{H} \alpha$, GOES and Yohkoh Hard X-ray Telescope (HXT) data sets. In Sect. 3 we analyse these distributions and quantify the effect of the $\mathrm{H} \alpha$ bias and also examine how it varies with size of the flare in terms of its emissions. We make our conclusions and note the implications for future work in Sect. 4.

\section{Longitude distributions of solar flares}

In this section we present histograms for the distribution of solar flares listed in three catalogues: $\mathrm{H} \alpha$ (two stations), GOES and Yohkoh HXT. The histograms count the number of flares above a minimum size in nine $10^{\circ}$ bins of longitude, without distinguishing between east and west. The uncertainty on each bin is estimated as the square root of the count on the assumption of Poisson statistics. For all the data, the longitudes used were taken from the catalogue in question, though in all cases the longitudes are measured from $\mathrm{H} \alpha$ flare positions.

\section{1. $H \alpha$}

We consider two stations that regularly report $\mathrm{H} \alpha$ flares: Holloman (USA: $106^{\circ} \mathrm{W}, 33^{\circ} \mathrm{N}$ ) and Learmonth (Australia: $\left.115^{\circ} \mathrm{E}, 21^{\circ} \mathrm{S}\right)$. We chose these as they offer complementary coverage from opposite hemispheres. In addition both sites have been able to report nearly every day. We use the data from Jan. 11994 to Dec. 31 2000, giving a total of 4573 flares for Holloman and 3531 flares for Learmonth.

Figure 1 plots the longitude histogram of all flares that appeared in the $\mathrm{H} \alpha$ catalogue for Holloman and Learmonth, including flares of importance "S" (Subflare) and greater and of brightness "F" (faint) and brighter. (The H $\alpha$ "importance" measures the spatial area of the flare varying from " $S$ " for compact events to " 4 " for the largest flares. The $\mathrm{H} \alpha$ brightness is a qualitative classification with three categories: "F" faint, "N" normal and " $\mathrm{B}$ " bright.) There is a tenth bin in the histogram corresponding to flares that were assigned longitudes of greater than or equal to $90^{\circ}$ where $\mathrm{H} \alpha$ emission is seen across the limb.

The data from both stations are consistent with a uniform distribution for longitudes less than $70^{\circ}$. Clearly Holloman reported more flares than Learmonth in total, though the numbers in the two bins at the limb become similar within uncertainties. This suggests that the bias is stronger for Holloman.

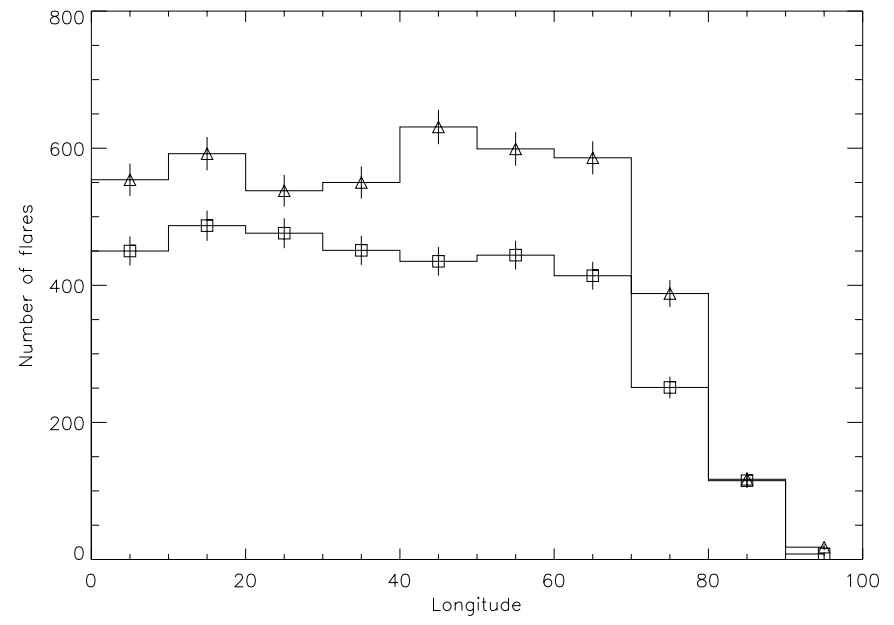

Fig. 1. The histograms for the longitude distribution of solar flares as observed in $\mathrm{H} \alpha$. The triangles are for Holloman and the squares are for Learmonth.

\subsection{GOES}

The histogram for the GOES data is shown in Fig. 2, for all flares with a GOES class "B" and greater. We use the data from Jan. 11991 to Dec. 31 2000, giving a total of 9745 flares. Notice that there is a tenth bin for longitudes greater than or equal to $90^{\circ}$ (remember that the longitude of the flare comes from the $\mathrm{H} \alpha$ flare location).

The distribution appears uniform for longitudes less than $60^{\circ}$, as compared to $70^{\circ}$ for the $\mathrm{H} \alpha$. Note that the GOES data-set is larger, resulting in smaller errors bars than was the case for the $\mathrm{H} \alpha$.

\section{3. $H X T$}

The HXT longitude histogram (Fig. 3) contains all flares that had a peak count rate of at least 10 counts/s/SC in the instrument's LO channel, the background typically being 1 count/s/SC. We use the data from Oct. 11991 to Feb. 21 2001, giving a total of 477 flares.

Given the small data-set, and so relatively larger uncertainties in comparison to $\mathrm{H} \alpha$ and GOES, it is more difficult to assign a longitude to the end of the uniform part of the distribution. However, it is somewhere above $60^{\circ}$, and so the HXT data is at least consistent with the GOES and the $\mathrm{H} \alpha$ distributions.

\section{Analysis}

\subsection{Comparing PDFs}

In order to compare the three distributions and so compare the strength of the bias in each, we plot the Probability Density Functions (PDFs) in Fig. 4. Each point on the PDF is obtained by taking the corresponding count from the histogram and dividing it by the bin size $\left(10^{\circ}\right)$ and the total number of events in the histogram.

The PDFs agree with each other to within uncertainties, and appear to be flat up to about $60^{\circ}$. For longitudes greater than about $70^{\circ}$ it is clear that the HXT PDF is significantly greater 


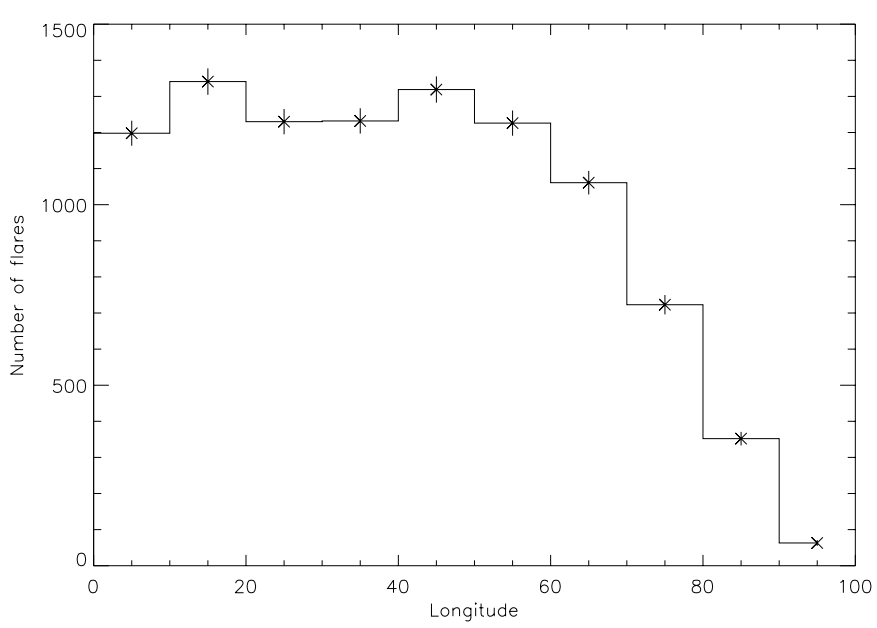

Fig. 2. The histograms for the longitude distribution of solar flares with GOES class greater than B.

than others. Greater than $80^{\circ}$ the GOES PDF is greater than that of the $\mathrm{H} \alpha$ ones, though this difference is less significant.

Table 1 contains the results of a $\chi^{2}$ comparison between all six different pairs of the four longitude distributions. The $\chi^{2}$ was calculated according to

$\chi^{2}=\sum_{i=1}^{10} \frac{\left(N_{1 i}-\alpha N_{2 i}\right)^{2}}{\left(N_{1 i}+\alpha^{2} N_{2 i}\right)}$

where $N_{1 i}$ and $N_{2 i}$ are the counts in histogram bin $i$ for data sets 1 and 2 respectively. Also, $\alpha=N_{1} / N_{2}$, the ratio of the total counts in sets 1 and 2, is used to normalise the histograms for comparison. This normalisation reduces the number of degrees of freedom to nine. A lack of significant difference will therefore produce $\chi^{2} \sim 9$. Also in the table is the probability of obtaining the given $\chi^{2}$ or greater. We can safely assume that the $\chi^{2}$ distribution is meaningful here because all bins of the histogram are well populated (the lowest count being 7), so that $N_{1 i}$ and $N_{2 i}$ both have a Poisson distribution that is Gaussianlike. Table 1 shows that all data-sets have $\chi^{2}>9$ with correspondingly small probabilities. We can therefore conclude that all distributions are significantly different to each other.

\subsection{Quantifying the $\mathrm{H}_{\alpha}$ bias}

The $\chi^{2}$ values tell us that there is a significant difference in the longitude distributions, but they do not tell us the strength of the $\mathrm{H} \alpha$ bias. So we define a bias index $\beta$ as

$\beta=\frac{8 N_{1}}{N_{\mathrm{d}}}$

where $N_{1}$ and $N_{\mathrm{d}}$ are the number of limb and disk flares respectively. We will use a longitude of $80^{\circ}$ to delineate limb from disk; Fig. 4 shows that all PDFs have decreased significantly at this longitude. Also, we omit all flares falling in the last histogram bin, i.e. all flares with longitude greater than or equal to $90^{\circ}$. So, if there is no bias, then $\beta$ should have a value of 1 , and

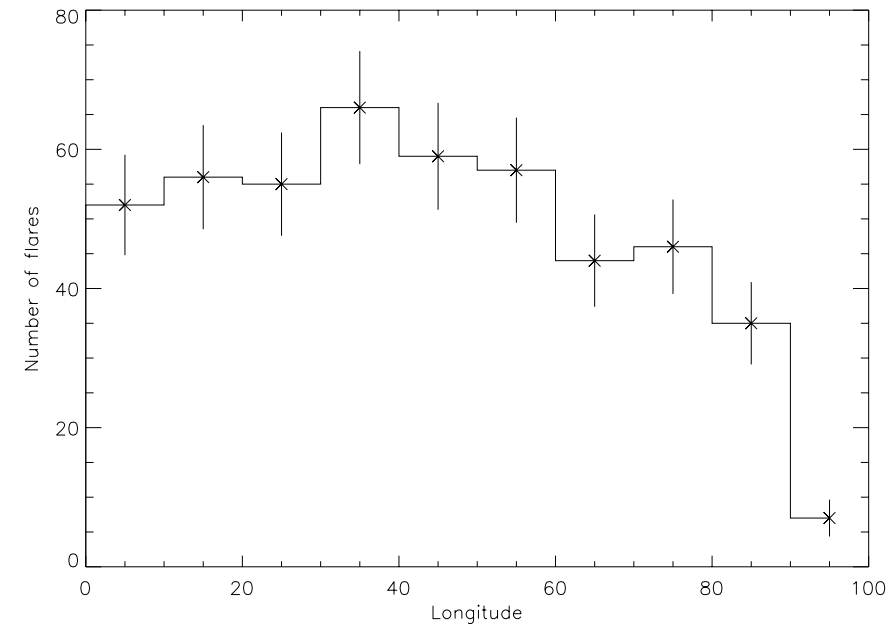

Fig. 3. The histograms for the longitude distribution of solar flares with Yohkoh HXT LO channel counts of 10 or more.

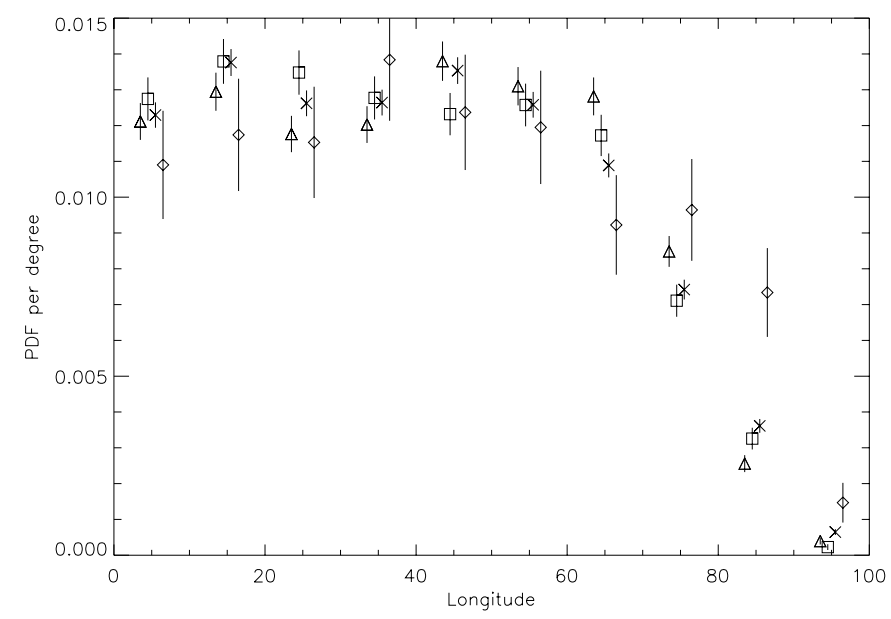

Fig. 4. The Probability Density Functions (PDFs) for $\mathrm{H} \alpha$ Holloman (triangles), Learmonth (squares), GOES (crosses) and HXT (diamonds). The points are offset from the bin centres (by multiples of 0.5 degrees) so that the error bars may be easily compared.

an $\mathrm{H} \alpha$ bias will result in $\beta<1$. Assuming Poisson statistics, we can express the uncertainty on $\beta$ as

$\sigma_{\beta}=\beta\left(\frac{1}{\sqrt{N_{\mathrm{l}}}}+\frac{1}{\sqrt{N_{\mathrm{d}}}}\right)$

Table 2 lists the numbers of limb and disk flares in each data set together with its bias index and uncertainty. The $\mathrm{H} \alpha$ data appear to have slightly different biases within the given uncertainties. The GOES bias index is several standard deviations greater than those for Holloman, but is comparable with that of the Learmonth. The HXT index is more tha two standard deviations greater than any of the others. It is worth noting that all indices overlap at the three sigma level.

The value of $\beta$ can be interpreted as the fraction of limb flares that have a location determined from $\mathrm{H} \alpha$ observations. Clearly, judging by Learmonth and Holloman, more than $70 \%$ of $\mathrm{H} \alpha$ limb flares are not identified. Given that the locations of GOES and HXT flares come from $\mathrm{H} \alpha$ it might seem surprising that the biases for these data sets are significantly different. 
Table 1. Comparison of solar flare longitude distributions using $\chi^{2}$ (9 degrees of freedom).

\begin{tabular}{llll}
\hline \hline Data 1 & Data 2 & $\chi^{2}$ & Probability \\
\hline H $\alpha$ Learmonth & H $\alpha$ Holloman & 23.0 & 0.0063 \\
GOES & H $\alpha$ Holloman & 35.3 & $5.4 \mathrm{E}-05$ \\
GOES & H $\alpha$ Learmonth & 21.3 & 0.011 \\
HXT & H $\alpha$ Holloman & 27.7 & 0.0011 \\
HXT & H $\alpha$ Learmonth & 25.4 & 0.0026 \\
HXT & GOES & 18.6 & 0.029 \\
\hline
\end{tabular}

Table 2. The numbers of limb ( $>80^{\circ}$ longitude) and disk flares, together with bias index $\beta$ and its one sigma uncertainty $\sigma_{\beta}$.

\begin{tabular}{lllll}
\hline \hline Data set & $N_{\mathrm{l}}$ & $N_{\mathrm{d}}$ & $\beta$ & $\sigma_{\beta}$ \\
\hline H $\alpha$ Holloman & 117 & 4438 & 0.211 & 0.023 \\
H $\alpha$ Learmonth & 115 & 3408 & 0.270 & 0.030 \\
GOES & 352 & 9330 & 0.302 & 0.019 \\
HXT & 35 & 435 & 0.644 & 0.140 \\
\hline
\end{tabular}

One possible explanation is that mild directivity effects (either from emission or absorption of radiation) might be countering the $\mathrm{H} \alpha$ bias; this is consistent with the fact that the HXT data set shows the least bias. Another possibility is that some kind of selection effect involving the brightness or size of a flare is affecting the contents of the different catalogues in different ways. To help discriminate between these two possibilities we now consider how the $\mathrm{H} \alpha$ bias is related to different measures of flare size.

\subsection{The $\mathrm{H} \alpha$ bias and flare size}

If we restrict the GOES data set to flares greater than class M5, and the $\mathrm{H} \alpha$ data to flares that have brightness $\mathrm{N}$ (Normal) or $\mathrm{B}$ (Bright), then the $\chi^{2}$ and $\beta$ values change to the ones given in Tables 3 and 4. We have not selected a subset of the larger HXT flares because this data set is already small, and its flares probably already correspond to the brighter flares in the GOES and $\mathrm{H} \alpha$ data sets. As evidence of this, $50 \%$ of HXT catalogue flares are $\mathrm{M}$ class or brighter as compared to only $10 \%$ of all GOES catalogue flares.

The GOES > M5 and HXT PDFs shown in Fig. 5 appear to be consistent within the uncertainties, and the $\chi^{2}$ and probability, as given in Table 3, are now 9.4 and 0.40 . In other words, there appears to be no significant difference between the GOES $>$ M5 and HXT longitude distributions. The bias index for GOES $>$ M5 is $\beta=0.657 \pm 0.095$, and is not significantly different from the HXT value of $\beta=0.644 \pm 0.14$.

One explanation for the lesser bias for HXT and GOES > M5 flares may be that larger, X-ray bright flares are also generally brighter in $\mathrm{H} \alpha$ and so more readily identified when near the limb. Figure 6 plots $\mathrm{H} \alpha$ importance and brightness against GOES brightness for the 475 flares in the HXT catalogue where both $\mathrm{H} \alpha$ and GOES information were given. The graphs show an apparent trend for larger X-ray flares to have larger $\mathrm{H} \alpha$ importances and brightnesses, though with significant scatter. Part of this scatter is undoubtedly due to the discreteness of the $\mathrm{H} \alpha$
Table 3. Comparison of solar flare longitude distributions for the brighter flares using a $\chi^{2}$ (9 degrees of freedom).

\begin{tabular}{llll}
\hline \hline Data 1 & Data 2 & $\chi^{2}$ & Probability \\
\hline H $\alpha$ Learmonth & $\mathrm{H} \alpha$ Holloman & 7.3 & 0.61 \\
goes & $\mathrm{H} \alpha$ Holloman & 55.4 & 0.0 \\
goes & $\mathrm{H} \alpha$ Learmonth & 27.8 & 0.0010 \\
hxt & $\mathrm{H} \alpha$ Holloman & 34.0 & $8.8 \mathrm{E}-05$ \\
hxt & $\mathrm{H} \alpha$ Learmonth & 17.5 & 0.041 \\
hxt & goes & 9.4 & 0.40 \\
\hline
\end{tabular}

Table 4. The numbers of brighter limb ( $>80^{\circ}$ longitude) and disk flares, together with bias index $\beta$ and its one sigma uncertainty $\sigma_{\beta}$.

\begin{tabular}{lllll}
\hline \hline Data set & $N_{\mathrm{l}}$ & $N_{\mathrm{d}}$ & $\beta$ & $\sigma_{\beta}$ \\
\hline $\mathrm{H} \alpha$ Holloman & 5 & 186 & 0.215 & 0.112 \\
$\mathrm{H} \alpha$ Learmonth & 5 & 219 & 0.183 & 0.094 \\
goes & 80 & 974 & 0.657 & 0.095 \\
hxt & 35 & 435 & 0.644 & 0.140 \\
\hline
\end{tabular}

properties, though much of it could well be due to intrinsic characteristics of flares.

Comparison of the $\mathrm{H} \alpha \beta$ values in Tables 2 and 4 do not suggest any lessening of the bias for the brighter flares in the $\mathrm{H} \alpha$ catalogue. This appears to invalidate the large-flare origin of the bias proposed above. A possible resolution is if many flares near the limb have their $\mathrm{H} \alpha$ brightness underestimated, possibly for the same observational (or even limb-darkening) effects that are responsible for so many being missed. However, this does not appear to be the case as the relative proportion of flares in categories F, N and B are the same (Table 5) to within uncertainties for both limb flares and disc flares. However this evidence is misleading because, if the underlying brightness distribution is power law, which is almost certainly the case, then a bias in $\mathrm{H} \alpha$ brightness will not affect the relative proportions of flares in the F, N and B categories (see Appendix A). We are therefore forced to look elsewhere for evidence of a lessening bias for brighter and/or larger $\mathrm{H} \alpha$ flares.

We can understand the difference in bias between the $\mathrm{X}$-ray and $\mathrm{H} \alpha$ data by looking to the data on the duration of flares in $\mathrm{H} \alpha$. Firstly, for flares in the HXT catalogue with positions identified in $\mathrm{H} \alpha$, the average duration of the flare given in the $\mathrm{H} \alpha$ Holloman and Learmonth catalogues are 8.7 min and $7.0 \mathrm{~min}$ respectively. The corresponding averages for all flares in these $\mathrm{H} \alpha$ catalogues are $5.0 \mathrm{~min}$ and $4.6 \mathrm{~min}$. According to the t-test these differences are significant with greater than $90 \%$ confidence. In other words, X-ray flares tend to be associated with longer $\mathrm{H} \alpha$ flares. Further, in Table 5 we can see that there are only about half as many $\mathrm{H} \alpha$ flares with greater than $10 \mathrm{~min}$ duration on the limb (longitude $>80^{\circ}$ ) as would be expected from the disk population. This tells us that the length of flares is often underestimated near the limb, and most probably that shorter duration flares can be missed altogether. This therefore gives us a possible explanation for the lesser bias for flares in the X-ray catalogues: flares producing X-rays are associated with longer $\mathrm{H} \alpha$ flares which are less likely to be missed at the limb. 


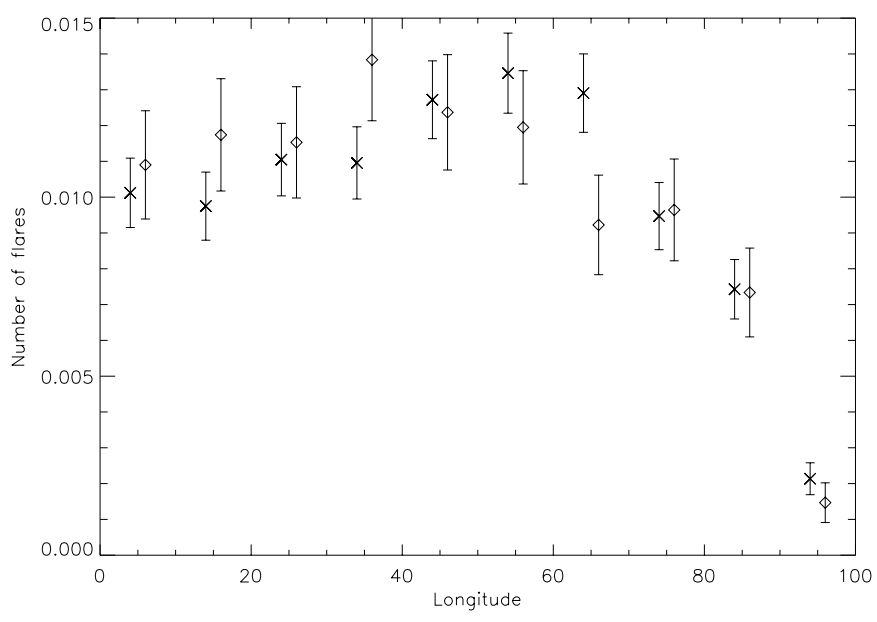

Fig. 5. The Probability Density Functions (PDFs) for GOES > M5 (crosses) and HXT (diamonds). The points are offset from the bin centres (all at multiples of 0.5 degrees) so that the error bars may be easily compared.

\section{Discussion}

A significant bias exists in longitude distributions constructed from catalogues of $\mathrm{H} \alpha$, GOES and Yohkoh HXT flares. The bias is greatest for $\mathrm{H} \alpha$ flares, with between $70 \%$ and $80 \%$ of limb flares (longitudes greater then $80^{\circ}$ ) missing from the catalogue. The bias is smaller for the X-ray flares, with about $35 \%$ of limb flares missing in the Yohkoh HXT catalogue. Since flare positions in the GOES and HXT catalogues are drawn from the $\mathrm{H} \alpha$ catalogues, it is clear that the bias originates in the identification of $\mathrm{H} \alpha$ flares.

The reasons for the differences in bias are less obvious. To investigate whether a selection effect acts against weak flares, we repeated our analysis of the bias for only the larger flares in the $\mathrm{H} \alpha$ (normal and bright classes) and GOES (larger than M5) catalogues. The restricted GOES flare set gave a decreased bias, identical to the HXT catalogue within uncertainties. However, the brighter $\mathrm{H} \alpha$ flares showed no such decrease. The reason for this contradictory result is most likely related to the coarseness of the $\mathrm{H} \alpha$ classes (discussed in more depth in Appendix A) and their apparently weak correlation with GOES class. To resolve the problem we considered the $\mathrm{H} \alpha$ flare duration, as it is a more quantifiable property of $\mathrm{H} \alpha$ flares. Unlike $\mathrm{H} \alpha$ brightness, the duration exhibits a clear bias, with the fraction of limb flares of duration greater than 10 min being half that for disk flares. It appears therefore that limb flares of shorter duration are more likely to be missed in $\mathrm{H} \alpha$ near the limb than on the disk, giving us a possible explanation for the bias.

Whether the bias in flare duration is the cause of the bias itself, or a statistical symptom of it cannot be determined from the data considered here. However, flare duration is correlated with flare size (in terms of emission), either because larger flares remain above some observable threshold for longer times, or because it is an intrinsic characteristic of flares themselves. Either way, a link was demonstrated between $\mathrm{H} \alpha$ flare duration and X-ray brightness. Flares appearing in the HXT catalogue have an average $\mathrm{H} \alpha$ duration that is 3 to 4 min longer
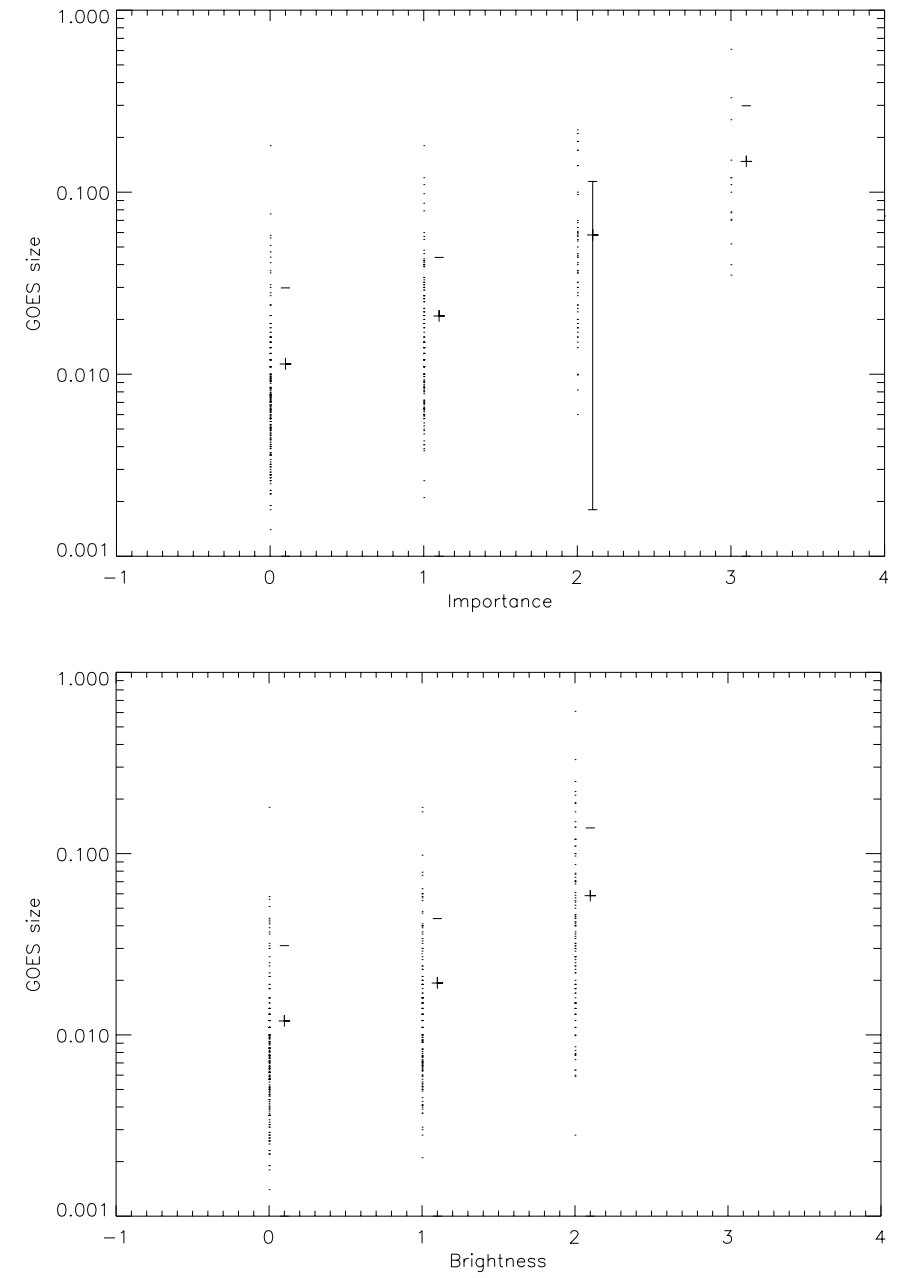

Fig. 6. GOES brightness (where $\mathrm{X} 1.0$ is 0.1 ) plotted against $\mathrm{H} \alpha$ importance ( $\mathrm{S}$ is 0 ) and brightness $(\mathrm{F}, \mathrm{N}, \mathrm{B}$ is $0,1,2)$ for 475 flares in the HXT catalogue. The "+" symbol marks the mean value and the upper one standard deviation position is marked with a "-". In all cases but one the lower one standard deviation can be regarded as zero.

Table 5. The fractions of flares in various categories for limb and disk subsets.

\begin{tabular}{lcccccc}
\hline \hline Limb/Disk & $F$ & $\mathrm{~N}$ & $\mathrm{~B}$ & Imp. $\geq 2$ & Dur. > 10 min. Total Flares \\
\hline \multicolumn{5}{c}{ Holloman } \\
Limb & 0.965 & 0.026 & 0.009 & 0.000 & 0.053 & 114 \\
Disk & 0.958 & 0.033 & 0.009 & 0.015 & 0.107 & 4573 \\
& \multicolumn{5}{c}{ Learmonth } \\
Limb & 0.930 & 0.050 & 0.020 & 0.020 & 0.040 & 100 \\
Disk & 0.936 & 0.050 & 0.014 & 0.015 & 0.100 & 3531 \\
\hline
\end{tabular}

than the average for all $\mathrm{H} \alpha$ flares. We can therefore conclude that the difference in bias between catalogues is due to a selection effect working against weaker and/or shorter duration limb flares.

An important implication of this work is that directivity studies on hard X-ray flares should rely on flare positions determined from hard X-ray images, and not from flare positions from $\mathrm{H} \alpha$ catalogues. If the latter method is used then the $\mathrm{H} \alpha$ 
bias will oppose the directivity bias which acts towards the limb. The directivity of hard X-ray emission ( 10-500 keV) is expected to be quite mild. For example, Li (1995) found that the directivity was $<1.5$ (see paper for the definition of this index) for a sample of 72 flares observed simultaneously by the Gamma Ray Spectrometer (GRS) and Hard X-ray Burst Spectrometer (HXRBS) on the Solar Maximum Mission for photon energies $<100 \mathrm{keV}$. In contrast, at $1 \mathrm{MeV}$ the directivity can be as high as 6.5. It is therefore conceivable that mild directivity at lower HXR energies could be completely countered by the $\mathrm{H} \alpha$ bias. The main motivation for investigating the hard X-ray directivity is to discover what kind of pitch angle distribution the electrons have. An isotropic distribution giving little directivity implies electrons are either accelerated this way, or have been collisionally evolved into this state. Strong directivity would indicate that electron beams are created with small pitch angles (presumably in the corona) and can propagate to the chromosphere relatively unaffected by collisions before they produce their Hard X-rays. In addition to pitch angle information, McTiernan \& Petrosian (1990) consider the many other factors involved in directivity which leads them to question the validity of certain common assumptions, notably that the magnetic field is always vertical and that electrons are always accelerated in the corona. A subsequent paper McTiernan \& Petrosian (1991) concludes that directivity arguments place limits on the amount of magnetic field convergence and suggest that flare electrons usually have beam-like pitch angle distributions. For all the above reasons, directivity information places constraints on both electron acceleration and transport in the solar atmosphere.

A possibility raised by the differing biases is that limb flares, especially those with their lower coronal emission being occulted, might be identified statistically in catalogues, without the use of images. This is desirable because it is much more time consuming to search through and construct images of flares than it is to search through catalogues, especially when dealing with tens of thousands of flares. It might also allow identification of limb flares with solar flares only observed by non-imaging instruments such as GOES, BATSE and Ulysses.

The most obvious indication that a flare is on the limb is that there is no $\mathrm{H} \alpha$ identification. However this is no guarantee of a limb flare, it could simply be that the flare was missed by the $\mathrm{H} \alpha$ observing network. Another possible indication of a limb flare is that although the flare was large in a non-imaged $\mathrm{X}$-ray observation, it was only observed as a small flare in $\mathrm{H} \alpha$. However, given the poor correlation between GOES class and $\mathrm{H} \alpha$ classes and the similarity of the F, N, B distribution for limb and disk, this method would almost certainly not work. In a similar way, it might be expected that partially occulted limb flares might have a large GOES class for its HXR brightness, especially at high energies. This is because high energy HXR emission is often confined to the chromosphere, whereas large soft X-ray bright loops extend above the limb into the corona. However, Fig. 7, which plots GOES brightness against HXT M1 (23-33 keV) counts shows that limb flares are not outliers on such a plot, though there may be a slight bias towards smaller hard X-ray brightness. This means that this method could not be used reliably either.

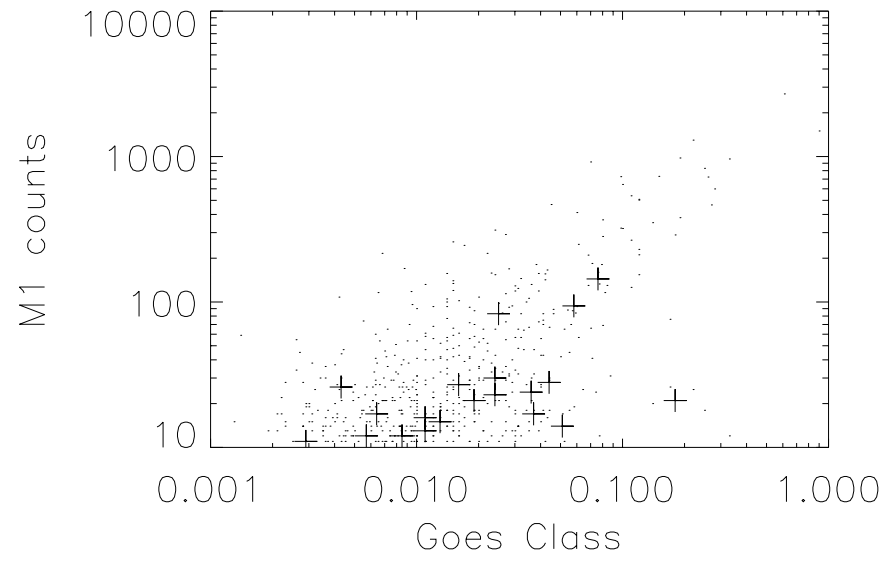

Fig. 7. GOES size (where X1.0 is 0.1) is plotted against HXT M1 counts. The points are all flares that had 10 counts or more in the HXT catalogue, and the "+" symbols mark flares that occurred at a longitude of $80^{\circ}$ or greater.

In practice, therefore, it does not seem that statistical properties of flares alone can identify limb flares. However, they can be used to select flares that might prove to be limb flares and worthy of further examination in an X-ray image. The method that we have used in another study is to first find flares listed in $\mathrm{X}$-ray catalogues (either soft or hard) that do not have an $\mathrm{H} \alpha$ identification. A search for an event occurring at the same time in the $\mathrm{H} \alpha$ catalogue is required to confirm this because sometimes $\mathrm{H} \alpha$ entries are missing in the X-ray catalogues. Once a bright X-ray flare with no $\mathrm{H} \alpha$ counter-part is found then confirmation that it is a limb flare requires either an X-ray image or, less conclusively, that a known active region was straddling the limb at that time.

Our conclusion is that the use of $\mathrm{H} \alpha$ positions in solar flare catalogues causes a bias away from the limb in the flare longitude distribution. The bias apparently becomes weaker when dealing with higher energy/shorter wavelength data sets. There are two competing interpretations of these results. The first, as discussed previously, is that larger flares (as measured by one or more of brightness, area or duration) are less often overlooked near the limb in $\mathrm{H} \alpha$ images. The alternative interpretation is that weak X-ray directivity makes it more likely that limb flares appear in the X-ray catalogues. The first interpretation naturally accounts for all our results except the lack of variation in the $\mathrm{H} \alpha$ brightness distribution between the limb and the disk, though this might simply be due to the crudeness of $\mathrm{H} \alpha$ brightness classification. The second interpretation does not have this problem, but it does require X-ray directivity effects at surprisingly low energies (1-20 keV). Also, directivity does not obviously explain why the longitude distribution of flares with GOES > M5 is indistinguishable from that of the HXT flares, whereas the two significantly differ if all GOES flares are included.

Acknowledgements. In this work we made use of data from the following sources: the Yohkoh satellite which is a project of the Institute of Space and Astronautical Science, Japan in collaboration with the U.S. and U.K.; NASA-GSFC, data from NOAA GOES; US Air Force Solar Optical Observing Network (SOON) - Holloman AFB, New Mexico; Learmonth Observatory, Australia. 


\section{Appendix A: The bias in $\mathrm{H} \alpha$ brightness}

In this Appendix we wish to explain the interesting result that the distribution of $\mathrm{H} \alpha$ flare brightness appears to be the same on the disk and near the limb. We do this by considering how the ratios of the number of flares in each category, e.g. $n_{B} / n_{N}$, varies as a function of longitude $\theta$. In particular we are interested in the assumptions that lead to such ratios being independent of $\theta$.

Let the brightness distribution of $\mathrm{H} \alpha$ flares, as measured in terms of a continuous value $f$, be given by $n(f)$. Also, let the probabilities of a flare of brightness $f$ and longitude $\theta$ being observed to be of brightness $f_{1}$ be given by the function $p\left(f_{1} \mid f, \theta\right)$. We can then write the observed distribution of flares as

$n_{1}\left(f_{1}, \theta\right)=\int_{0}^{\infty} p\left(f_{1} \mid f, \theta\right) n(f) \mathrm{d} f$.

We can now make two assumptions, the validity of which we will discuss later. 1) $p\left(f_{1} \mid f, \theta\right)=\delta\left(f-f_{1} / h(\theta)\right)$, which means that a flare of brightness $f$ at longitude $\theta$ is always recorded as being of (lesser) brightness $f_{1}=h(\theta) f$. 2) The distribution of solar flare brightnesses is power law, i.e. $n(f)=n_{0} f^{-\alpha}$. Under these two assumptions, the observed flare distribution can be written as

$n_{1}\left(f_{1}, \theta\right)=n_{0} h^{\alpha-1}(\theta) f_{1}^{-\alpha}$.

At this point it is clear that ratios of $n_{1}$ at different values of $f_{1}$ will be independent of $\theta$. The same is true for ratios of integrals, such as the cumulative distribution function $N_{1}\left(f_{1}\right)=$ $\int_{f_{1}}^{\infty} n_{1} \mathrm{~d} f_{1}$. Thus, under the above two assumptions, we can conclude that taking ratios, such as the number of $N$ and $B \mathrm{H} \alpha$ flares, cannot provide evidence of a longitude bias.

However, there is no reason to suppose that the particular functional form for $p$ introduced in assumption 1 is true. If we consider flares of a particular observed size $f_{1}$, we can see that the preceding theory (A.2) predicts that the longitude PDF varies as $h^{\alpha-1}(\theta)$, irrespective of the value of $f_{1}$. The differing longitude PDFs (Fig. 4) for $\mathrm{H} \alpha$, GOES and HXT data sets (which in this order represent flares of increasing magnitude) do not agree with this prediction and so argue against the validity of assumption 1, at the very least suggesting that $h$ is a function of $f$ as well as $\theta$.

There are however alternative explanations of why there is no evidence of a variation of longitude bias with $\mathrm{H} \alpha$ brightness. One explanation involving X-ray directivity is discussed at the end of Sect. 4. Another is based on the fact that $\mathrm{H} \alpha$ brightness relies on the subjective inspection of a photograph. It is therefore reasonable to suppose that $\mathrm{H} \alpha$ brightness classification can often place flares of similar $f$ in different classes. This is saying that the function $p\left(f_{1} \mid f, \theta\right)$ has a non-zero width in $f_{1}$ (as opposed to the dirac delta function used above) and that this width is comparable to or greater than the width of the $\mathrm{H} \alpha$ brightness categories F, N and B. The effect of the longitude bias can then be regarded as a displacement of the peak of $p\left(f_{1} \mid f, \theta\right)$ to an $f_{1}$ less than the true brightness of the flare $f$, with the displacement increasing as $\theta$ increases (with a possible change in widths too). The point is that the large, possibly overlapping widths might be masking the evidence of how the longitude bias changes with $f$. In effect, the mis-classification together with the particular definitions of brightness categories could explain why we fail to detect the $\mathrm{H} \alpha$ flare brightness's effect on the longitude distribution.

\section{References}

Bumba, V., Garcia, A., \& Klvana, M. 2000, Sol. Phys., 196, 403

Kane, S., Anderson, K. A., Evans, W. D., Klebesadel, R. W., \& Laros, J. 1979, ApJ, 233, 151

Kane, S., Fenimore, E. E., Klebesadel, R. W., \& Laros, J. 1988, ApJ, 326, 1017

Kosugi, T. 1985, PASJ, 37, 575

Li, P. 1995, ApJ, 443, 855

Masuda, S., Kosugi, T., Tsuneta, S., \& Hara, H. 1996, Adv. Space Res., 17, 63

Miller, J. A., Cargill, P. J., Holman, G. D, et al. 1997, JGR, 102, 1463

McTiernan, J. M., \& Petrosian, V. 1990, ApJ, 359, 541

McTiernan, J. M., \& Petrosian, V. 1991, ApJ, 379, 381

Ozguc, A., \& Altas, L. 1996, Sol. Phys., 163, 327

Petrosian, V. 1985, ApJ, 299, 987

Vestrand, W. T., Forrest, D. J., Chupp, E. L., Rieger, E., \& Share, G. H. 1986, Adv. Space Res., 6, 123 